Communications in Physics, Vol.23, No. 1 (2013), pp. 67-73

\title{
DETERMINATION OF WAVELENGTH SHIFT OF FIBER BRAGG GRATING SENSORS BY TUNABLE SINGLE-MODE DIODE LASER
}

\author{
NGUYEN THE ANH, PHAM THANH SON, NGUYEN THUY VAN, HOANG THI \\ HONG CAM, NGO QUANG MINH, BUI HUY, AND PHAM VAN HOI \\ Institute of Materials Science, VAST
}

\begin{abstract}
We propose a novel principle of determination of fiber Bragg grating (FBG) wavelength shift which is impacted by a variation of physical parameters such as temperature, pressure and/or strain. In common case the wavelength shift of FBG was monitored by wavelength measurement using a high-cost spectrometer and a broad band light source. In our proposed technique the wavelength shift of FBG can be determined by change of lasing wavelength of distributed feedback laser (DFB-laser) due to the change of laser substrate temperature. The maximal opto-electrical intensity of photodetector would be obtained when the laser wavelength and FBG reflection wavelength are coincided. The FBG sensor prototype has shown excellent response for laser temperature change in the range of $10^{0} \mathrm{C}-50^{0} \mathrm{C}$ with the ratio $\Delta \lambda / \Delta T$ of the DFB laser is of $77.5 \mathrm{pm} . \mathrm{K}^{-1}$. Key features of the proposed technique are fabrication of low-cost FBG sensors for civil engineering.
\end{abstract}

\section{INTRODUCTION}

Optical sensors based on fiber Bragg gratings (FBG) have attracted considerable attention since the early stage of their discovery. FBG sensors have become widely known and researched among the devices in the photonics community and their utilization and commercial growth rised intensively. Given the capability of FBGs to detect the multitude of parameters such as strain, temperature, pressure, chemical and biological agents and many others coupled with their flexibility of design to be used as single point or multipoint sensing arrays and their relative low cost, make them ideal devices to be adopted for a multitude of different sensing applications and implemented in different fields and industries [1]. FBG sensors have advantages in many aspects, for instance, immunity to electromagnetic interference, compact size, light-weight, flexibility, high operation stability, high temperature tolerance, and resistance to harsh environment, very low optical insert loss, narrow bandwidth wavelength reflection, and linearity in response over many orders of magnitude and compatibility with the existing fiber optic system. FBG sensors can be used in measurement of many physical parameters such as strain [2], vibration [3], pressure [4] and temperature [5,6]. It is apparent that any shift in the Bragg wavelength is influenced by the temperature, pressure and/or strain factors. For example, the thermal response of temperature sensors based on FBG is induced due to both the inherent thermal expansion of the fiber material and the temperature dependence of the refractive index. The shift of the FBG wavelength caused due to physical parameters often detects by wavelength measurement. It means a disadvantage of FBG sensors is the necessary 
of measuring a wavelength shift by spectrometer that makes the high-cost of FBG-based sensor apparatus.

In this paper we propose a new principle of determination of FBG wavelength shift by tuning the lasing wavelength of DFB single-mode laser due to the change of laser substrate temperature. The maximal optoelectrical intensity of detector would be obtained when the DFB lasing wavelength and FBG reflection wavelength are coincided. The sensor prototype has shown excellent response for measuring temperature of ambient in the range of $0^{\circ} \mathrm{C}-100^{\circ} \mathrm{C}$ when the laser substrate temperature changed in the range of $10^{\circ} \mathrm{C}-50^{\circ} \mathrm{C}$ and the ratio $\Delta \lambda / \Delta \mathrm{T}$ of the $\mathrm{DFB}$ laser is of $77.5 \mathrm{pm} . \mathrm{K}^{-1}$.

\section{PRINCIPLE}

The Bragg grating wavelength $\left(\lambda_{B}\right)$ is the center wavelength of the light reflected from a Bragg grating, that depends upon the effective refractive index of the fiber core $\left(n_{e f f}\right)$ and the periodicity of the Bragg grating $(\Lambda)$ through the relation $\lambda_{B}=2 n_{e f f} \Lambda$. The effective index of the fiber core as well as the periodical spacing between the grating planes will be affected by ambient temperature, pressure and strain. The fractional FBG wavelength shifts for a temperature change $\Delta T$ and pressure $\Delta \mathrm{P}$ caused by the changes in the grating spacing and the effective index may be written as follow [7]:

$$
\frac{\Delta \lambda}{\lambda_{B}}=[(\alpha+\xi)] \Delta T+\left[-\frac{1-2 \nu}{E}+\frac{n_{e f f}^{2}}{2 E}(1-2 \nu)\left(2 p_{12}+p_{11}\right)\right] \Delta P
$$

where: $\alpha=(1 / \Lambda)(\partial \Lambda / \partial T)$ is the thermal expansion coefficient $\left(0.55 \times 10^{-6} .{ }^{\circ} \mathrm{C}^{-1}\right.$ for silica), and $\xi=(1 / n)(\partial n / \partial T)$ is the thermo-optic coefficient, which is of $8.6 \times 10^{-6} .{ }^{\circ} \mathrm{C}^{-1}$, $\nu$ is Poisson's ratio, $\mathrm{p}_{11}$ and $\mathrm{p}_{12}$ are the components of the strain-optic tensor, $\mathrm{E}$ is modulus of the elasticity for a germanium- doped silica fiber. The schematic diagram of wavelength shift measurement using photodiode is shown in Fig. 1.

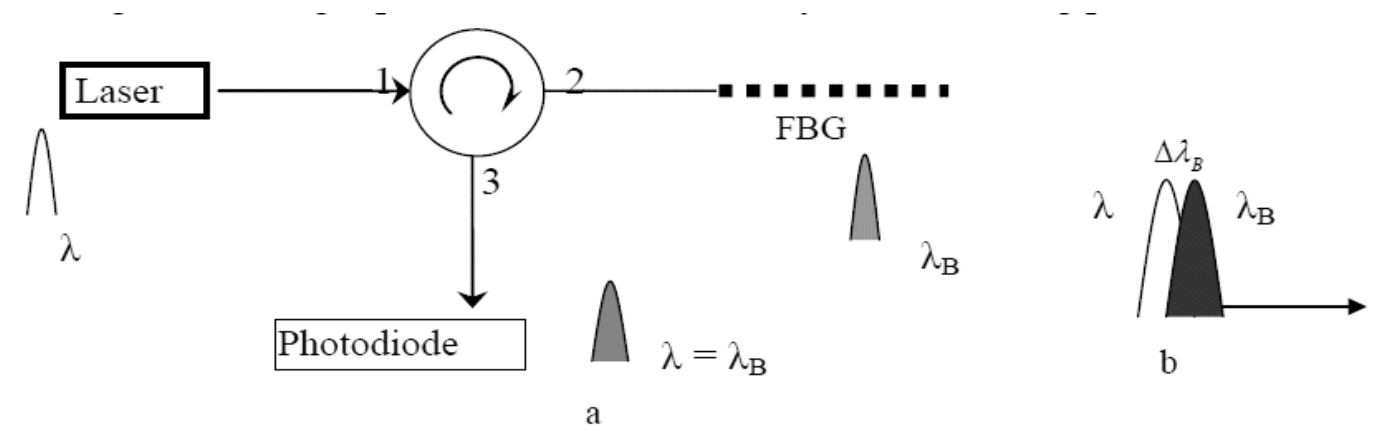

Fig. 1. Principle schematic diagram of wavelength shift measurement using photodiode

We intend to compare the change of reflection Bragg wavelength, which shifts due to physical parameters, and adjustment of emission wavelength of single-mode laser by controlling temperature of laser substrate. Fig.1 shows emission wavelength of single-mode laser connected to gate 1 of circulator device and laser beam propagates via gate 2 to fiber Bragg grating, which has the FBG reflection wavelength equaled to laser wavelength at 
room temperature. The reflection light from FBG is collected by photodiode at gate 3 . At the first step, if the FBG was not influenced by environmental impacts such as the change of temperature and pressure, so photodiode detected the maximum power at wavelength $\lambda$ emitted from the laser (Fig. 1a). When the FBG is influenced by environmental impacts, the light reflected from Bragg grating shifts its wavelength of a value $\Delta \lambda_{B}$ in comparison to laser wavelength (see Fig. 1b). In this case the photo-electrical intensity of photodiode would be decreased and reaches to 0 when $\Delta \lambda_{B}$ is large [8]. The opto-electrical intensity strongly depends on the ratio of the incident intensity and reflected intensity from FBG. The intensity of reflection light from FBG expressed by a modified Gaussian function as follows [5]:

$$
\mathrm{I}_{r e f}=\mathrm{R}_{F B G} \mathrm{I}_{\text {in }} \exp \left(-\Delta \lambda^{2} / \Delta \lambda_{0}^{2}\right)
$$

where, $\mathrm{R}_{F B G}$ is reflectivity of $\mathrm{FBG} ; \mathrm{I}_{i n}$ is incoming light intensity; $\Delta \lambda_{0}$ is bandwidth of signal spectrum and $\Delta \lambda$ is spacing between Bragg wavelength center $\lambda_{B}$ and signal wavelength $\lambda$. Calculation ratio between reflection and incoming intensities is shown in Fig. 2. The reflection intensity is maximal when the wavelength spacing between incoming and reflection is zero.

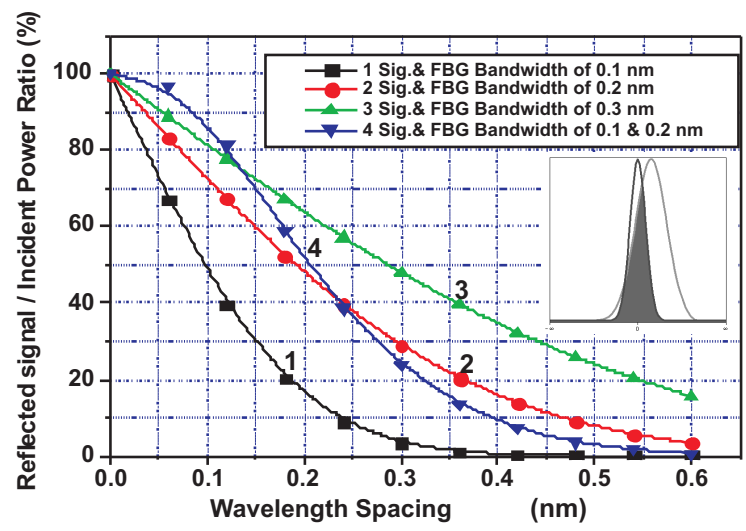

Fig. 2. Dependence of reflection intensity upon the spectral linewidth of incoming signal and wavelength spacing between reflection and incident light. Inset: Crosssection of incoming and reflection spectra

In principle, we can control the emitted wavelength from laser by changing the temperature of laser substrate. If the wavelength shifts of lasing emission by temperature $\Delta \lambda_{L}$ are equaled to FGB wavelength shift $\left(\Delta \lambda_{B}\right)$, so the photo-electrical intensity of photodiode becomes maximal again. That means we can measure the physical impact of environment by the adjustment of temperature of laser substrate without using the high-cost spectrometer.

\section{EXPERIMENT AND RESULTS}

In our work, the FBG was fabricated by holographic method using a KrF-Excimer laser emitted at wavelength of $248 \mathrm{~nm}$ and Talbot interferometer. The optical fiber was a 
commercial photosensitive germano-silicate single mode fiber. We can make single and/or array of FBG with different Bragg wavelengths into one optical fiber. The Bragg wavelength at room temperature was in the range of 1530-1550 $\mathrm{nm}$ and a reflectivity was of 75-90\% with full-width-half-maximum (FWHM) bandwidth of $0.15-0.30 \mathrm{~nm}$. The length of FBG was of $15 \mathrm{~mm}$. The broad-band light source used for characterization of FBG was an Amplified Spontaneous Emission (ASE) from Erbium-doped Fiber Amplifier (EDFA). The spectral measurement was performed with a reflection scheme using 1550nm-fiber optic circulator. The reflection spectrum was observed with an Optical Spectrum Analyzer (Advantest Q8384) with wavelength resolution of $0.01 \mathrm{~nm}$. For testing the characteristics of FBG depended on the ambient temperature, we use thermal box which can control the temperature from $0^{\circ} \mathrm{C}$ to $110^{\circ} \mathrm{C}$ with changed step of $5^{\circ} \mathrm{C}$. The shift of testing FBG reflection wavelength depended on the temperature is shown in figure 3. The Bragg wavelength of FBG is of $1550.45 \mathrm{~nm}$ at room temperature $\left(23^{\circ} \mathrm{C}\right)$. When we reduce the temperature to $10^{\circ} \mathrm{C}$, the $\mathrm{FBG}$ wavelength reflection is $1549.61 \mathrm{~nm}$. If we increase temperature of box to $100^{\circ} \mathrm{C}$, the $\mathrm{FBG}$ wavelength reflectivity is $1551.8 \mathrm{~nm}$.

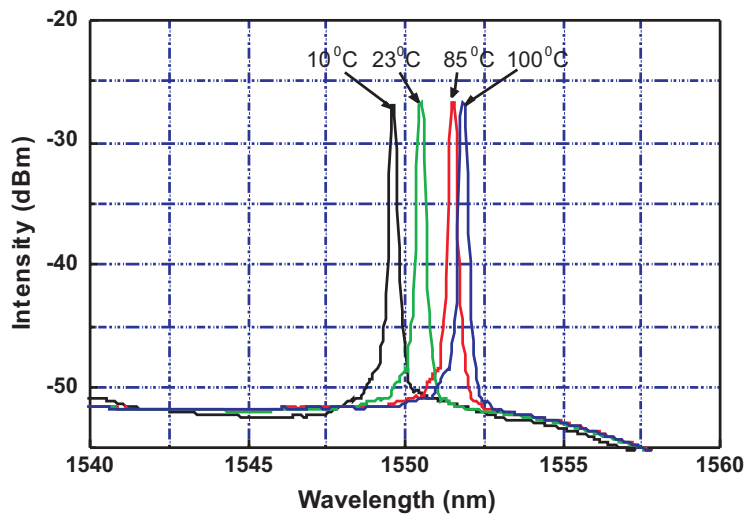

Fig. 3. Wavelength shift of FBG reflection wavelength due to temperature change from $10^{\circ} \mathrm{C}$ to $100^{\circ} \mathrm{C}$

The FBG can be recoated by different thermal-sensitive materials such as Teflon, polymer and metals [9] and embedded on the Copper $(\mathrm{Cu})$ substrate. We should choice the embedded FBG with polymer (silicone) which has a reflection wavelength at room temperature matching with the emission wavelength of laser diode. The dependence of the silicone-embedded FBG wavelength shift upon the ambient temperature, which is shown in Fig. 4. It shows a non-linear dependence in the range of $0^{\circ} \mathrm{C}-110^{\circ} \mathrm{C}$. That means for the measurement of ambient temperature by embedded FBG sensors we should have the data base of wavelength shift of FBG in the concrete temperature range. At temperature range of $0^{\circ} \mathrm{C}-110^{\circ} \mathrm{C}$ the silicone-embedded Bragg wavelength shift is of 3.0 $\mathrm{nm}$ and the average temperature sensitivity of the FBG corresponds to $27.2 \mathrm{~nm} . \mathrm{K}^{-1}$.

A distributed feedback laser (DFB) is a type of laser diode, quantum cascade laser or optical fiber laser where the active region of the device is sandwiched between two diffraction gratings which provides optical feedback for the laser. The grating is constructed so as to reflect only a narrow band of wavelengths, and thus produce a single 


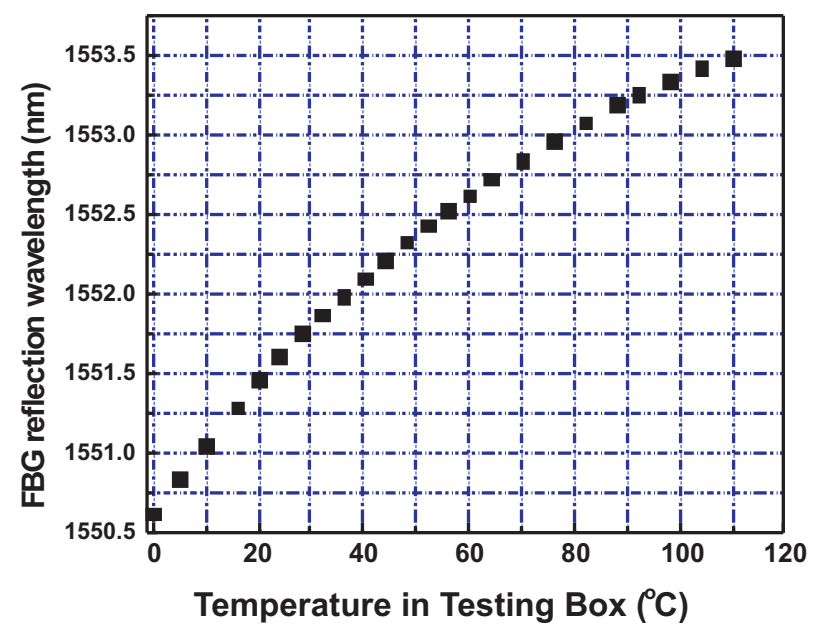

Fig. 4. Dependence of the FBG wavelength shift on the ambient temperature

longitudinal lasing mode. Altering the laser substrate temperature causes a change of the semiconductor bandgap, refractive index and thermal expansion of grating. A change in the refractive index and thermal expansion of the grating alter the wavelength selection of the grating structure and thus the wavelength of the laser output, producing a wavelength tunable laser. In this work, we use DFB single-mode laser 2A0469 made by a Photonics company. The spectral linewidth and maximal output power of emission are of $0.2 \mathrm{~nm}$ and $5 \mathrm{~mW}$, respectively. For testing tunable of lasing wavelength depended on the laser substrate temperature we use Peltier cooler which can adjust exactly the temperature change of $0.2^{\circ} \mathrm{C}$ in range of $10^{\circ} \mathrm{C}-50^{\circ} \mathrm{C}$. The dependence of lasing wavelength shift and lasing intensity of laser diode upon substrate temperature have been shown in figure 5 .

In the temperature range of $10^{\circ} \mathrm{C}-50^{\circ} \mathrm{C}$ the dependence of emitted wavelength shift upon the altering temperature is linear and the temperature sensitivity of laser emitted wavelength was of $77.5 \mathrm{pm} . \mathrm{K}^{-1}$. In our experiment the lasing intensity of laser diode decreased on $50 \%$ when the laser substrate temperature increased from $25^{\circ} \mathrm{C}$ to $50^{\circ} \mathrm{C}$. It is remarkable, that when we combine the temperature sensitive of FBG wavelength and laser diodes, we can build configuration of FBG sensors that can measure the ambient temperature from $0^{\circ} \mathrm{C}$ to $100^{\circ} \mathrm{C}$.

Table 1 presents the combination of FBG wavelength shift in the temperature range of $0^{\circ} \mathrm{C}-100^{\circ} \mathrm{C}$ and temperature of laser substrate in the range of $10^{\circ} \mathrm{C}-50^{\circ} \mathrm{C}$ with the photoelectrical maximal intensity of photodiode. The accuracy of measured photoelectrical signal is of $+/-0.02 \mathrm{~V}$. The photoelectrical maximal intensity of photodiode is of about $2.05+/-0.02 \mathrm{~V}$, when the laser wavelength and FBG reflection wavelength are coincided in the temperature range $0-30^{\circ} \mathrm{C}$ and it would be decreased when the laser temperature is more than $30^{\circ} \mathrm{C}$. In experiment we obtained measurement accuracy of $+/-0.3^{\circ} \mathrm{C}$ for temperature sensors. It is remarkable that the FBG wavelength shift due to physical impacts of ambient can be measured by the change of laser substrate temperature without 


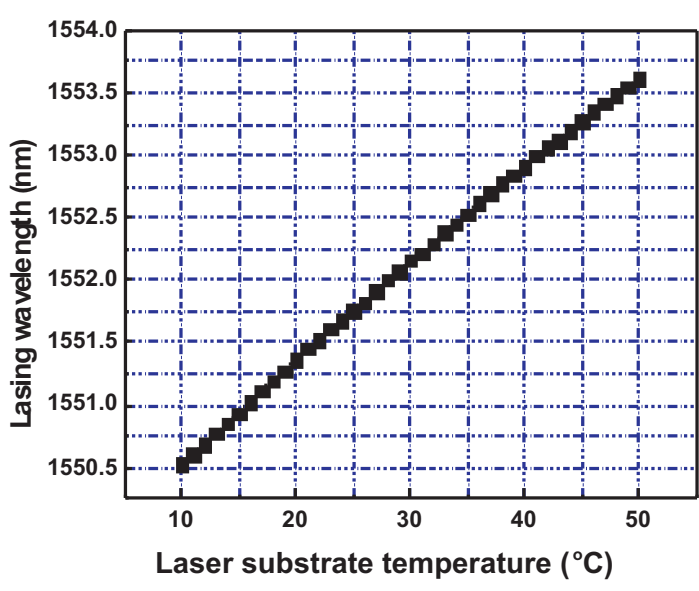

(a)

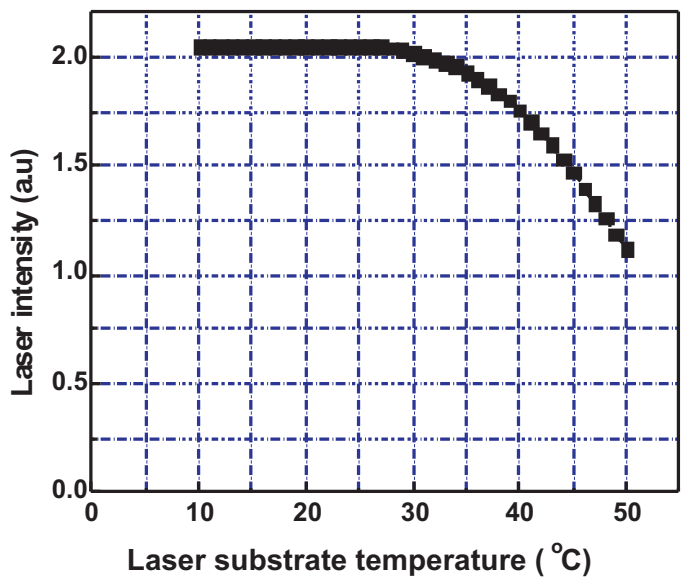

(b)

Fig. 5. Dependence of emission wavelength (a) and lasing intensity (b) of laser diode on the laser substrate temperature

Table 1. Maximal signal intensity of Photodiode versus temperature change of FBG and laser diode

\begin{tabular}{ccccc}
\hline $\begin{array}{c}\text { Temp. in Box } \\
\text { with FBG }\left({ }^{\circ} \mathrm{C}\right)\end{array}$ & $\begin{array}{c}\text { FBG wavelength } \\
\lambda_{B}(\mathrm{~nm})\end{array}$ & $\begin{array}{c}\text { Temp. of } \\
\text { Laser }\left({ }^{\circ} \mathrm{C}\right)\end{array}$ & $\begin{array}{c}\text { Lasing wavelength } \\
\lambda_{L}(\mathrm{~nm})\end{array}$ & $\begin{array}{c}\text { Maximal intensity } \\
\text { of PD (Volt) }\end{array}$ \\
\hline 0 & 1550.620 & 10.94 & 1550.61 & 2.07 \\
10 & 1551.056 & 16.2 & 1551.06 & 2.07 \\
20 & 1551.610 & 22.6 & 1551.61 & 2.05 \\
30 & 1551.826 & 25.8 & 1551.84 & 2.03 \\
40 & 1552.096 & 29.2 & 1552.10 & 2.02 \\
50 & 1552.276 & 31.8 & 1552.30 & 1.97 \\
60 & 1552.626 & 35.9 & 1552.58 & 1.90 \\
70 & 1552.846 & 39.0 & 1552.85 & 1.81 \\
80 & 1553.056 & 41.7 & 1553.05 & 1.67 \\
90 & 1553.236 & 44.5 & 1553.21 & 1.51 \\
100 & 1553.376 & 46.3 & 1553.27 & 1.47 \\
\hline
\end{tabular}

use of spectrometer, if we have a data base of the FBG wavelength shift due to the physical parameters and lasing wavelength shift due to the temperature change.

\section{CONCLUSION}

We proposed a novel principle of determination of FBG wavelength shift by using tunable single-mode laser diode. Our FBG sensor prototype has the responsible ratio $\Delta \lambda / \Delta T$ of wavelength change versus temperature in the range of $10^{0}-50^{\circ} \mathrm{C}$ of the DFB laser and the temperature sensitivity of silicone-embedded FBG in the range of $0^{\circ} \mathrm{C}$ $-100^{\circ} \mathrm{C}$ are of $77.5 \mathrm{pm} . \mathrm{K}^{-1}$ and $27.2 \mathrm{pm} . \mathrm{K}^{-1}$, respectively. It results the measurement accuracy of $\pm 0.3^{\circ} \mathrm{C}$ in the case of temperature sensors. In general, it can change the 
temperature of laser substrate to match the single-mode emission wavelength with the silicone-embedded FBG reflection wavelength, in dependence of the environment parameters that allows to determinate the small FBG wavelength shift of some nanometers. That means the proposed sensor can apply in measurement of FBG wavelength shift without use of high-cost spectrometer. We believe that the new FBG sensors have a big potential for application in the civil engineering.

\section{ACKNOWLEDGMENTS}

This work is financially supported by Institute of Materials Science, Vietnam Academy of Science and Technology. This work had been using the apparatus of National Key Laboratory for Electronic Materials and Devices in Institute of Materials Science.

\section{REFERENCES}

[1] Q.Y. Chen, and P. Lu, Atomic, Molecular and Optical Physics, Ed. by L.T. Chen, Nova Sci. Publisher Inc., pp. 235-260 (2008)

[2] D.C. Betz, G. Thursby, B. Culshaw, and W.J. Staszewski, IEEE J. Light. Technol. 24 (2006) 10191026

[3] X. Dong, Y. Huang, K. Lang, W. Zhang, G. Kai, and X. J. Dong, Microw. Opt. Tech. Lett. 42 (2004) 474-476.

[4] B. Peng, Y. Zhao, J. Yang, and M. Zhao, Measurement 38 (2005) 176-180

[5] G. M. H. Flockhart, R. R. J. Maier, J. S. Barton, W. N. MacPherson, J. D. C. Jones, K. E. Chisholm, L. Zhang, I. Bennion, I. Read, and P. D. Foote, Appl. Opt. 43 (2004) 2744-2751

[6] J. Jung, H. Nam, B. Lee, J.O. Byun, and N.S. Kim, Appl. Opt. 38 (1999) 2752-2754

[7] G. Meltz and W. W. Morey, Proc. SPIE, v.1516 (1991) 185-199

[8] T. T. Nguyen, T. H. Do, V. H. Pham, T. B. Pham, X. V. Ha, T. T. H. Chu, Asean J. on Sci. Es Technol. for Development 24 (1\&2) (2007) 41-46

[9] V. H. Pham, T. B. Pham, T. T. A. Pham, X. V. Ha, T. T. H. Chu, T. T. Nguyen, J. Sensors $\mathcal{G}^{2}$ Actuators A: Physical 141 (2008) 334-338

Received 21 December 2012. 